\title{
Molecular analysis of pediatric brain tumors identifies microRNAs in pilocytic astrocytomas that target the MAPK and NF-kB pathways
}

Tania A. Jones ${ }^{1 \dagger}$, Jennie N. Jeyapalan ${ }^{1 \dagger}$, Tim Forshew ${ }^{1,6}$, Ruth G. Tatevossian ${ }^{1,7}$, Andrew R. J. Lawson ${ }^{1,8}$, Sheena N. Patel ${ }^{1}$, Gabriel T. Doctor', Muhammad A. Mumin'1, Simon R. Picker ${ }^{2,3}$, Kim P. Phipps ${ }^{4}$, Antony Michalski ${ }^{5}$, Thomas S. Jacques ${ }^{2,3^{*}}$ and Denise Sheer ${ }^{1 *}$ (D)

\begin{abstract}
Introduction: Pilocytic astrocytomas are slow-growing tumors that usually occur in the cerebellum or in the midline along the hypothalamic/optic pathways. The most common genetic alterations in pilocytic astrocytomas activate the ERK/MAPK signal transduction pathway, which is a major driver of proliferation but is also believed to induce senescence in these tumors. Here, we have conducted a detailed investigation of microRNA and gene expression, together with pathway analysis, to improve our understanding of the regulatory mechanisms in pilocytic astrocytomas.

Results: Pilocytic astrocytomas were found to have distinctive microRNA and gene expression profiles compared to normal brain tissue and a selection of other pediatric brain tumors. Several microRNAs found to be up-regulated in pilocytic astrocytomas are predicted to target the ERK/MAPK and NF-KB signaling pathways as well as genes involved in senescence-associated inflammation and cell cycle control. Furthermore, IGFBP7 and CEBPB, which are transcriptional inducers of the senescence-associated secretory phenotype (SASP), were also up-regulated together with the markers of senescence and inflammation, CDKN1A (p21), CDKN2A (p16) and IL1B.

Conclusion: These findings provide further evidence of a senescent phenotype in pilocytic astrocytomas. In addition, they suggest that the ERK/MAPK pathway, which is considered the major driver of these tumors, is regulated not only by genetic aberrations but also by microRNAs.
\end{abstract}

Keywords: Grade I astrocytoma, Children, Senescence-associated secretory phenotype (SASP), miR-146a, miR-155, Neuroinflammation, KIAA1549-BRAF

\section{Introduction}

Pilocytic astrocytomas (WHO grade I) are the most common central nervous system tumors in the 5 to 19 year age group. They are cystic, well-circumscribed tumors, which rarely progress and can usually be removed using surgery [9]. This accounts for their having

\footnotetext{
* Correspondence: t.jacques@ich.ucl.ac.uk; d.sheer@qmul.ac.uk

${ }^{\dagger}$ Equal contributors

2Department of Histopathology, Great Ormond Street Hospital for Children NHS Trust, London WC1N 3JH, UK

${ }^{1}$ Centre for Genomics and Child Health, Blizard Institute, Barts and The London School of Medicine and Dentistry, Queen Mary University of London, 4 Newark Street, London E1 2AT, UK

Full list of author information is available at the end of the article
}

a more favourable prognosis than diffuse and other infiltrative astrocytomas [39]. Molecular studies on pilocytic astrocytomas have identified recurrent $B R A F$ gene fusions and other alterations that activate the ERK/ MAPK signal transduction pathway $[15,27,56]$. Activated ERK/MAPK signaling is believed to drive cellular proliferation and then to trigger senescence, giving rise to the indolent phenotype of pilocytic astrocytomas $[24,42]$.

MicroRNAs are short RNA molecules that modulate gene expression post-transcriptionally by repressing translation or degrading the mRNA transcript [26]. They regulate numerous biological processes including 
cell proliferation and differentiation, and have major roles in embryogenesis, including brain and spinal cord development and neurogenesis [13, 41]. Many microRNAs are involved in the initiation and progression of cancer [3, 37], and recent studies of pediatric brain tumors have identified tumor-specific microRNA signatures $[5,21,38]$.

To further our knowledge of the molecular drivers of pilocytic astrocytomas, we conducted a detailed investigation of microRNA and gene expression in these tumors, and characterised the findings by pathway analysis. Our results revealed a distinctive microRNA and gene expression profile in pilocytic astrocytoma compared to other pediatric brain tumors. We found that many predicted targets of up-regulated microRNAs in pilocytic astrocytomas are known regulators of the ERK/MAPK and NF- $\mathrm{B}$ pathways. These findings suggest an important modulatory role for microRNAs in critical pathways involved in pilocytic astrocytomas.

\section{Materials and methods}

Tumor samples and controls

miRNA and mRNA profiling was performed on a range of fresh frozen brain tumor samples (the test cohort) from 57 children, aged 1-16 years, and on 7 normal adult brain controls (4 frontal lobe and 3 cerebellum, Biochain) (Table 1a). A further 13 pediatric brain tumor samples were used as a validation cohort (Table 1b). Tumors were classified using criteria defined by the World Health Organization (WHO) [36]. Access to samples and clinical data complied with Local Research Ethics Committee (LREC) regulations: Great Ormond Street Hospital LREC reference number 05/Q0508/153. All the test pilocytic astrocytomas were shown previously to contain the KIAA1549-BRAF gene fusion [32]. The BRAF V600E mutation was also examined in the high-grade astrocytomas in the test cohort. KIAA1549$B R A F$ fusion and the $B R A F$ V600E mutations were also tested in the validation cohort using PCR according to published protocols [32, 45].

\section{MicroRNA and gene expression arrays}

MicroRNA expression was profiled in the test tumor cohort and the normal brain controls using Illumina MicroRNA Expression Arrays (MI-v2). This system screens 1,146 known and putative microRNAs including $97 \%$ of microRNAs in miRBase (Release 12.0). Gene expression profiles were obtained for the same tumors and controls using the Illumina HumanHT-12_v3 Beadchip system. RNA was extracted from the samples using TRIzol (Invitrogen) and eluted into RNAse-free water using the RNeasy mini kit (Qiagen). cDNA was synthesized using random hexamers and the SuperScript First-Strand cDNA synthesis system (Invitrogen). Hybridization and scanning were performed in-house at the Barts and The London Genome Centre.

\section{Statistical analysis}

The microRNA and gene expression-averaged tumor profiles were compared to normal adult brain controls using GenomeStudio and GeneSpring Multi-Omic Analysis v12.1 software. Data were subjected to thresholding, $\log$ transformation ( $\log 2)$, normalization (quantile) and baseline transformation (median to all samples). Normalized data of microRNA and gene expression are shown in Additional file 1: Table S1 and Additional file 2: Table S2. All data were deposited in NCBI's Gene Expression Omnibus [12] (http://www.ncbi.nlm.nih.gov/geo) and are accessible through GEO accession number GSE42658. Differential expression was defined as fold change $(\mathrm{FC})>2$ with FDR corrected (Benjamini Hochberg) $p$-values $<0.05$. Unsupervised hierarchical clustering (Euclidean method), was performed on both the microRNA and mRNA expression data. The differentially expressed microRNAs and genes are listed in Additional file 3: Table S3 and Additional file 4: Table S4 for tumor groups with more than three samples.

\section{Pathway analysis}

Ingenuity Pathway Analysis (Ingenuity Systems Inc., Redwood City, USA) was used to identify over-represented pathways for significantly differentially expressed genes in pilocytic astrocytomas. The significance value associated with the functional analysis is expressed as a $p$-value calculated by comparing the number of differentially expressed genes that participate in a given function or pathway, relative to the total number of occurrences of these proteins in all functional/pathway annotations stored in Ingenuity Pathway Knowledge Base. Multiple-testing corrected $p$-values were calculated using the Benjamini-Hochberg method. For each functional annotation, a statistical quantity is calculated called the regulation z-score. The purpose of the z-score is to identify increased or decreased biological functions that are likely implicated by the observed gene expression changes. Hence, the $p$-values measure the observed and predicted regulated gene sets, and the $\mathrm{z}$-score assesses the match of the observed and predicted up/down regulation patterns. For this study, we only considered a predicted activation/inhibition status as significant if the $p$-value was $<+/-0.05$ and the $\mathrm{z}$-score $>2.0$ or $<2.0$ respectively.

\section{Taqman microRNA assays}

TaqMan miRNA assays were used to validate differentially expressed microRNAs of interest. Control microRNAs RNU48 and miR-423-3p were tested for stability over all tumors and miR-423-3p was selected as the most stable control. Hence all samples were normalized 
Table 1 Pediatric tumor cohorts

\begin{tabular}{|c|c|c|c|c|c|c|}
\hline Tumor & Pathology & Grade & Location & Sex & Age & BRAF status \\
\hline \multicolumn{7}{|l|}{ a. } \\
\hline PGNT & Papillary glioneuronal & । & Frontal lobe & M & 14 & nd \\
\hline PA1 & Pilocytic astrocytoma & । & Posterior fossa & $\mathrm{F}$ & 16 & KIAA1549-BRAF exon 16-exon 9 \\
\hline PA2 & Pilocytic astrocytoma & । & Posterior fossa & M & 12 & KIAA1549-BRAF exon 16-exon 9 \\
\hline PA3 & Pilocytic astrocytoma & । & Posterior fossa & M & 14 & KIAA1549-BRAF exon 16-exon 9 \\
\hline PA5 & Pilocytic astrocytoma & । & Posterior fossa & $\mathrm{F}$ & 3 & KIAA1549-BRAF exon 16-exon 9 \\
\hline PA6 & Pilocytic astrocytoma & । & Cerebellum & $\mathrm{F}$ & 8 & KIAA1549-BRAF exon 15-exon 9 \\
\hline PA7 & Pilocytic astrocytoma & । & Cerebellum & M & 1 & KIAA1549-BRAF exon 16-exon 11 \\
\hline PA8 & Pilocytic astrocytoma & । & Cerebellum & M & 1 & KIAA1549-BRAF exon 16-exon 9 \\
\hline PA9 & Pilocytic astrocytoma & । & Cerebellum & $\mathrm{F}$ & 2 & KIAA1549-BRAF exon 16-exon 11 \\
\hline PA10 & Pilocytic astrocytoma & । & Cerebellum & M & 2 & KIAA1549-BRAF exon 16-exon 9 \\
\hline PA11 & Pilocytic astrocytoma & । & Cerebellum & M & 6 & KIAA1549-BRAF exon 16-exon 9 \\
\hline PA12 & Pilocytic astrocytoma & । & Cerebellum & $\mathrm{F}$ & 11 & KIAA1549-BRAF exon 15-exon 9 \\
\hline PA13 & Pilocytic astrocytoma & । & Posterior fossa & M & 4 & KIAA1549-BRAF exon 16-exon 9 \\
\hline PA14 & Pilocytic astrocytoma & । & Posterior fossa & $\mathrm{F}$ & 6 & KIAA1549-BRAF exon 16-exon 9 \\
\hline PA15 & Pilocytic astrocytoma & । & Posterior fossa & M & 9 & KIAA1549-BRAF exon 16-exon 9 \\
\hline DA1 & Diffuse astrocytoma & $\|$ & Temporal lobe & $\mathrm{F}$ & 12 & No BRAFV600E \\
\hline DA2 & Diffuse astrocytoma & $\|$ & Unknown & $\mathrm{F}$ & 14 & No BRAFV600E \\
\hline DA3 & Diffuse astrocytoma & $\|$ & Occipital lobe & M & 4 & nd \\
\hline DA4 * & Diffuse astrocytoma & $\|$ & Unknown & M & 17 & BRAFV600E present \\
\hline AA1 & Anaplastic astrocytoma & III & Conus medullaris & M & 15 & nd \\
\hline AA2 & Anaplastic astrocytoma & III & Temporal lobe & $\mathrm{F}$ & 3 & No BRAFV600E \\
\hline $\mathrm{AA} 3 *$ & Anaplastic astrocytoma & III & Unknown & $\mathrm{F}$ & 11 & No BRAFV600E \\
\hline GBM1 & Glioblastoma & IV & Frontal lobe & $\mathrm{F}$ & 15 & No BRAFV600E \\
\hline GBM2 & Glioblastoma & IV & Temporoparietal & M & 10 & No BRAFV600E \\
\hline GBM3 & Glioblastoma & IV & Brainstem & $\mathrm{F}$ & 4 & nd \\
\hline GBM4 & Glioblastoma & IV & Left frontal lobe & $\mathrm{F}$ & 10 & No BRAFV600E \\
\hline GBM5 & Glioblastoma & IV & Spinal cord & $\mathrm{F}$ & 7 & nd \\
\hline E1 & Ependymoma & III & Posterior fossa & M & 4 & \\
\hline E2 & Ependymoma & III & Parietal lobe & $\mathrm{F}$ & 6 & \\
\hline E3 & Ependymoma & $\|$ & Posterior fossa & $\mathrm{F}$ & 2 & \\
\hline E4 & Ependymoma & III & Posterior fossa & $\mathrm{F}$ & 2 & \\
\hline E5 & Ependymoma & $\|$ & Temporal lobe & M & 12 & \\
\hline E6 & Ependymoma & III & Posterior fossa & M & 6 & \\
\hline E7 & Ependymoma & III & Posterior fossa & M & 2 & \\
\hline E8 & Ependymoma & $\|$ & Supratentorial (frontal) & M & 14 & \\
\hline E9 & Ependymoma & $\|$ & Posterior fossa & M & 3 & \\
\hline E10 & Ependymoma & $\|$ & Posterior fossa & M & 4 & \\
\hline E11 & Ependymoma & III & Posterior fossa & $\mathrm{F}$ & nd & \\
\hline E12 & Ependymoma & III & Fourth ventricle & M & 1 & \\
\hline E13 & Ependymoma & $\|$ & Spinal seed & $\mathrm{F}$ & 6 & \\
\hline E14 & Ependymoma & III & Posterior fossa & $\mathrm{F}$ & 6 & \\
\hline M1 & Medulloblastoma & IV & Posterior fossa & M & 4 & \\
\hline M2 & Medulloblastoma & IV & Posterior fossa & M & 1 & \\
\hline
\end{tabular}


Table 1 Pediatric tumor cohorts (Continued)

\begin{tabular}{|c|c|c|c|c|c|c|}
\hline M3 & Medulloblastoma & IV & Posterior fossa & $\mathrm{F}$ & 6 months & \\
\hline M4 & Medulloblastoma & IV & Posterior fossa & M & 7 & \\
\hline M5 & Medulloblastoma & IV & Posterior fossa & M & 3 & \\
\hline M6 & Medulloblastoma & IV & Posterior fossa & M & 9 & \\
\hline M7 & Medulloblastoma & IV & Posterior fossa & M & 7 & \\
\hline M8 & Medulloblastoma & IV & Posterior fossa & M & 9 & \\
\hline M9 & Medulloblastoma & IV & Posterior fossa & M & 4 & \\
\hline $\mathrm{M} 10$ * & Medulloblastoma & IV & Posterior fossa & $\mathrm{F}$ & 5 & \\
\hline R1 & Atypical Rhabdoid & IV & Brainstem & M & 1 & \\
\hline R2 & Atypical Rhabdoid & IV & Posterior fossa & $\mathrm{F}$ & 7 months & \\
\hline R3 & Atypical Rhabdoid & IV & Supratentorial & $\mathrm{F}$ & 2 & \\
\hline R4 & Atypical Rhabdoid & IV & Posterior fossa & M & 9 months & \\
\hline R5 & Atypical Rhabdoid & IV & Posterior fossa & $\mathrm{F}$ & 2 months & \\
\hline P1 & Choroid Plexus Papilloma & । & Intraventricular (frontal lateral ventricle) & M & 10 months & \\
\hline P2 & Choroid Plexus Papilloma & I & Posterior fossa & M & 1 & \\
\hline P3 & Choroid Plexus Papilloma & I & Left lateral ventricle & M & 3 & \\
\hline P4 & Choroid Plexus Papilloma & I & Unknown & $\mathrm{F}$ & nd & \\
\hline $\mathrm{AC1}$ & Adult control cerebellum & & Cerebellum & M & 22 & \\
\hline AC2 & Adult control frontal lobe & & Frontal lobe & M & 41 & \\
\hline AC3 & Adult control cerebellum & & Cerebellum & M & 21 & \\
\hline AC4 & Adult control cerebellum & & Cerebellum & M & 26 & \\
\hline AC5 & Adult control frontal lobe & & Frontal lobe & M & 82 & \\
\hline AC6 & Adult control frontal lobe & & Frontal lobe & M & 25 & \\
\hline AC7 & Adult control frontal lobe & & Frontal lobe & M & 27 & \\
\hline \multicolumn{7}{|l|}{ b. } \\
\hline PA16 & Pilocytic astrocytoma & । & Posterior fossa & M & 9 & nd \\
\hline PA17 & Pilocytic astrocytoma & 1 & Posterior fossa & M & 12 & nd \\
\hline PA18 & Pilocytic astrocytoma & । & Suprasellar & M & 3 & KIAA1549-BRAF exon 15-exon 9 \\
\hline PA19 & Pilocytic astrocytoma & । & $3^{\text {rd }}$ ventricle & M & 16 & nd \\
\hline PA20 & Pilocytic astrocytoma & I & Brain stem & $\mathrm{F}$ & 3 & KIAA1549-BRAF exon 15-exon 9 \\
\hline PA21 & Pilocytic astrocytoma & I & Posterior fossa & $\mathrm{F}$ & 4 & KIAA1549-BRAF exon 16-exon 9 \\
\hline PA22 & Pilocytic astrocytoma & I & Posterior fossa & M & 16 & KIAA1549-BRAF exon 16-exon 9 \\
\hline PA23 & Pilocytic astrocytoma & I & Posterior fossa & $\mathrm{F}$ & 2 & KIAA1549-BRAF exon 16-exon 11 \\
\hline PA24 & Pilocytic astrocytoma & I & Posterior fossa & $\mathrm{F}$ & 12 & KIAA1549-BRAF exon 16-exon 9 \\
\hline GBM6 & Glioblastoma & IV & Midbrain/Thalamus & $\mathrm{F}$ & 14 & - \\
\hline GBM7 & Glioblastoma & IV & Occipital lobe & M & 4 months & - \\
\hline GBM8 & Glioblastoma & IV & Thalamic/intraventriclar & $\mathrm{F}$ & 10 & - \\
\hline GBM9 & Glioblastoma & $\|$ & Thalamic & M & 15 & - \\
\hline
\end{tabular}

(a) Test cohort analysed using Illumina MicroRNA Expression Arrays (MI-v2) and Illumina HumanHT-12 v3 Beadchip system, with the exception of samples marked with an asterisk $(*)$, that were used for validation only

(b) Validation cohort used for confirmation of differentially expressed microRNAs and genes. Annotation: - negative, nd not determined

to miR-423-3p and fold change calculated relative to the average expression in adult cerebellum and frontal lobe (BioChain, A508112 and B210079 respectively). Differential expression was validated for miR-542-3p, miR-503, miR146a, miR-34a, miR-155, miR-124\%, miR-129 and miR-
129*. TargetScan Release 6.2 (http://www.targetscan.org) was used to search for predicted targets of microRNAs of interest. MicroRNAs are named using the 'miR' prefix and a unique identifying number which is assigned sequentially [2]. Identical or very similar miRNA sequences within a 
species can be given the same number, with their genes distinguished by a letter and/or numerical suffixes (e.g. miR-450a and miR-450b are slightly different in sequence, whereas those of miR-450a-1 and miR-450a-2 are identical). Some miRNA hairpin precursors give rise to two excised miRNAs, one from each arm. Previous annotations used the nomenclature miR-124 and miR-124* for the guide and passenger strand respectively, and some of these names were included in our Illumina MicroRNA Expression Arrays (MI-v2). However, the mature sequences derived from both arms of the hairpin may be biologically functional, so current nomenclature uses miR-542-5p and miR-542-3p to designate miRNAs from the ' 5 ' and ' 3 ' arms respectively, [18, 29].

\section{RT-qPCR}

PCR primers were designed using Primer 3 software (http://frodo.wi.mit.edu/primer3/) (Additional file 5: Table S5). RT-qPCR was used to quantify the levels of mRNA expression for selected genes, using SYBR Green JumpStart Taq ReadyMix kit (Sigma-Aldrich), $50 \mathrm{ng} \mathrm{cDNA}$, and $0.1 \mu \mathrm{M}$ primers in a reaction volume of $20 \mu$ l. Assays were run on an ABI 7500 Real Time PCR System (ABI). PCR analyses were conducted in triplicate for each sample and melting curves analyzed for correct product size. Control genes TBP and CREB1 were tested for stability over all tumors, and TBP selected as the most stable endogenous control. Therefore, all samples were normalized to $T B P$ and fold change calculated relative to the average expression in adult cerebellum (BioChain, A508112), and frontal lobe (BioChain B210079). Relative quantification was calculated using the 2-ddC $\mathrm{C}_{\mathrm{t}}$ method [35].

\section{Results}

\section{Characterization of the pediatric brain tumor cohort}

We first examined microRNA and gene expression in the test tumor cohort that consisted of a papillary glioneuronal tumor $(n=1)$, pilocytic astrocytomas $(n=14)$, diffuse astrocytomas $(n=3)$, anaplastic astrocytomas $(n=2)$, glioblastomas $(n=5)$, atypical teratoid rhabdoid tumors (AT/RT) $(n=5)$, medulloblastomas $(n=9)$, ependymomas $(n=14)$ and choroid plexus papillomas $(n=4)$, total $=57$ samples (Table 1a). Differential microRNA expression across the averaged tumor groups was calculated to obtain fold changes compared to normal adult brain controls (FDR $<0.05$, Additional file 3: Table S3). The number of up- and down-regulated microRNAs for each tumor type is shown in Table 2. Unsupervised hierarchical clustering (Euclidean method) of microRNAs clustered the tumors by pathology in the low-grade tumors, and showed higher-grade tumors to be more widely distributed (Fig. 1a). A similar pattern was also found using gene expression data (Additional file 2: Table S2),
Table 2 Number of differentially expressed microRNAs in pediatric brain tumors compared to normal adult brain

\begin{tabular}{|c|c|c|c|c|}
\hline \multirow[t]{2}{*}{ Pathology } & \multirow[t]{2}{*}{$n$} & \multicolumn{2}{|c|}{ Regulation } & \multirow[t]{2}{*}{ Total } \\
\hline & & Up & Down & \\
\hline Pilocytic astrocytoma & 14 & 42 & 49 & 91 \\
\hline Pilocytic astrocytoma ${ }^{a}$ & 14 & 27 & 33 & 60 \\
\hline Diffuse astrocytoma & 3 & 1 & 2 & 3 \\
\hline Anaplastic astrocytoma & 2 & 6 & 8 & 14 \\
\hline Glioblastoma & 5 & 0 & 5 & 5 \\
\hline Ependymoma & 14 & 60 & 75 & 135 \\
\hline Medulloblastoma & 9 & 25 & 35 & 60 \\
\hline Medulloblastoma ${ }^{a}$ & 9 & 10 & 29 & 39 \\
\hline Atypical Teratoid/Rhabdoid tumor & 5 & 15 & 17 & 32 \\
\hline Choroid plexus papilloma & 4 & 47 & 63 & 110 \\
\hline
\end{tabular}

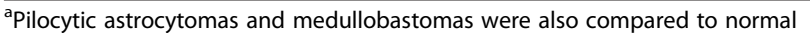
adult cerebellum. Fold change $>2$ with a FDR corrected $p$-value $<0.05$

with higher-grade tumors showing greater heterogeneity than low-grade tumors (Fig. 1b). Following initial comparison with other tumor types, microRNA profiles were then investigated in detail in pilocytic astrocytomas, all of which contained the KIAA1549:BRAF gene fusion.

\section{MicroRNA profile and the predicted targeted pathways in Pilocytic Astrocytomas}

Profiles from pilocytic astrocytomas were compared with combined adult brain controls, and also with normal adult cerebellum, since this subset of tumors were all located in the cerebellum (Additional file 3: Table S3). Up- and down-regulated microRNAs compared to normal adult cerebellum are shown in Table 3. Amongst the top upregulated microRNAs was a cluster located on Xq26.3, consisting of miR-542-5p, miR-542-3p, miR-503, mir-450a and miR-450b-5p (Fig. 2). The results were not genderbiased. Other top up-regulated microRNAs included miR224, miR-146a, miR-34a and the miR-106a miR-363 cluster (Table 3).

All the pilocytic astrocytomas used in the test cohort contain the KIAA1549-BRAF fusion that activates the ERK/MAPK pathway. Analysis of up-regulated microRNAs using TargetScan revealed several predicted targets to be regulators of the MAPK pathway. These included miR-155, which targets KRAS, miR-34a, which targets MAP2K1 (MEK1), and miR-503, which targets MAPK1 (ERK1) (Fig. 3). Other up-regulated microRNAs, including MiR-450b, miR-542-3p and miR-155, are all predicted to target ETS1, MYC and FOS, which are regulated by MAPK pathway components. In addition, the up-regulated microRNAs, miR-155, miR-199a, miR-21 and miR-146a (Fig. 4a), target genes within the NF-kB network (Figs. 3). Analysis of brain-specific microRNAs [49] showed no significant differences in expression in 


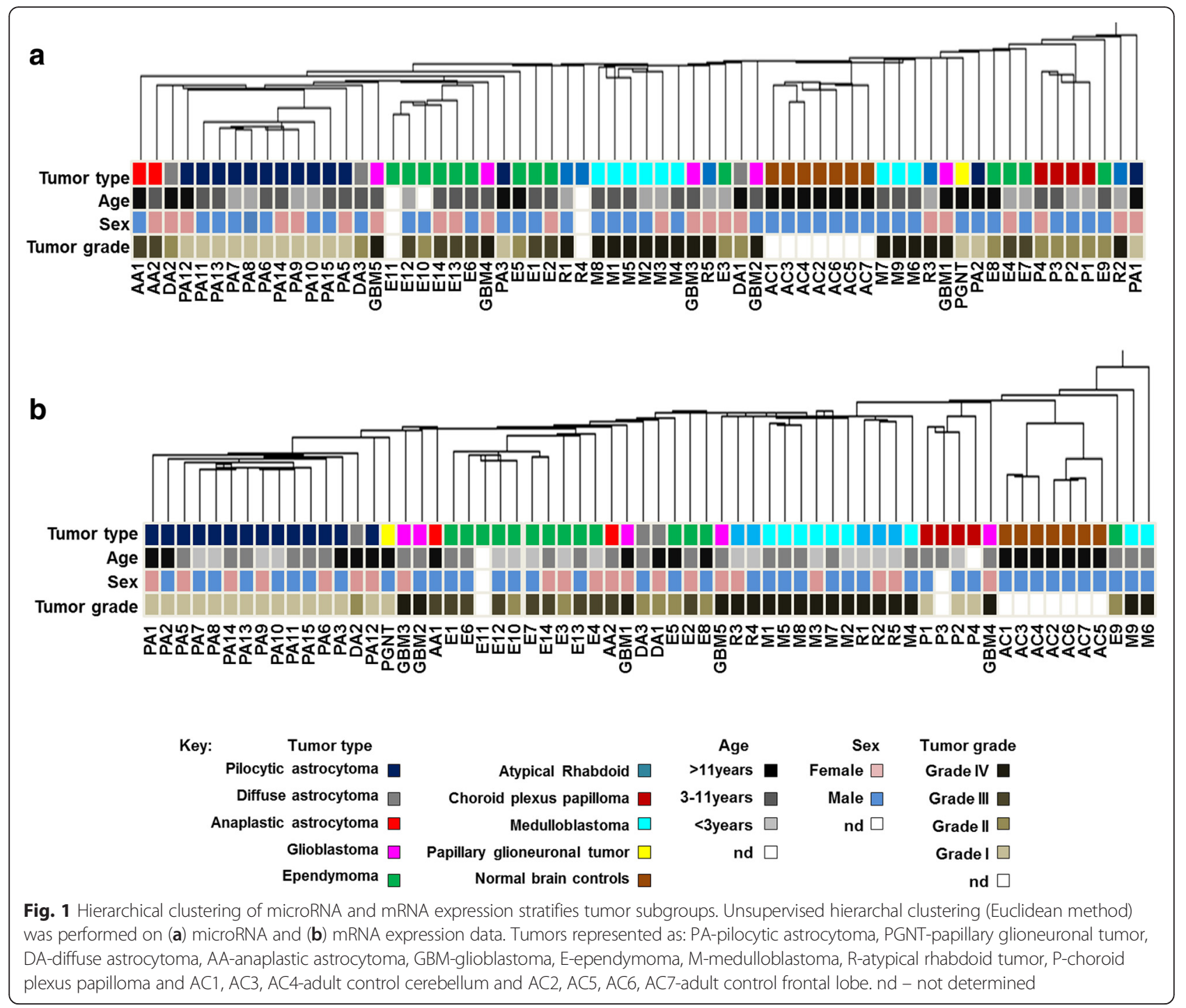

pilocytic astrocytomas, apart from down-regulation of miR-218. However, the majority of pilocytic astrocytomas showed low expression of neuronal-specific microRNAs, miR-129, miR-124 and miR-128 [50, 51] (Additional file 3: Table S3).

Oncogene-induced senescence is associated with activation of an inflammatory transcriptome [31], and since senescence markers have been identified in pilocytic astrocytomas, we examined expression levels of microRNAs and genes known to be associated with senescence. MiR-146a was amongst the top up-regulated microRNAs in pilocytic astrocytomas, and is known to modulate senescence-associated inflammatory mediators (Fig. 4a) [4]. Furthermore, CDKN1A ( $p 21)$ and CDKN2A (p16) were found to be up-regulated, together with the major transcriptional inducers of senescence-associated secretory phenotype (SASP), IGFBP7 and TIMP1 (Fig. 4b), as well as CEBPB, TIMP2, TIMP3, $A X L$ and other insulin- like growth factor (IGF)-binding proteins that stimulate inflammation, including IGFBP2, IGFBP3, IGFBP4 and IGFBP5 (Additional file 4: Table S4).

Ingenuity Pathway Analysis was used to compare gene expression data between pilocytic astrocytomas and normal adult cerebellum. The top canonical pathways showing significant up-regulation included Antigen Presentation Pathway, Graft-versus-Host Disease Signaling, Type I Diabetes Mellitus Signaling, Hepatic Fibrosis/Hepatic Stellate Cell Activation and Dendritic Cell Maturation. Analysis of the predicted activation state of up-stream transcription factors showed 70 factors predicted as 'activated' and 28 predicted as 'inhibited' $(p<0.05$, activation $z$-score $>2.0)$ (Additional file 6: Table S6a, b). Predicted 'activated' transcription factors included $N F-k B$ complex, TP53, IRF7, $C E B P B$, JUN, RELA and CEBPA, whilst predicted inhibited' transcription factors included TRIM24, MYC, MYCN, estrogen receptor and $R B$. 
Table 3 The top deregulated microRNAs in pilocytic astrocytoma compared to normal adult cerebellum

\begin{tabular}{|c|c|c|c|}
\hline TargetID & $\mathrm{p}$ (Corr) & FC & $\log F C$ \\
\hline \multicolumn{4}{|l|}{ a. } \\
\hline hsa-miR-224 & 0.00632 & 10.09 & 3.33 \\
\hline hsa-miR-542-5p & 0.00558 & 9.69 & 3.28 \\
\hline hsa-miR-542-3p & 0.02366 & 7.51 & 2.91 \\
\hline hsa-miR-450b-5p & 0.00285 & 6.89 & 2.78 \\
\hline hsa-miR-503 & 0.00120 & 6.80 & 2.76 \\
\hline hsa-miR-450a & 0.01211 & 6.67 & 2.74 \\
\hline hsa-miR-886-5p & 0.00551 & 6.17 & 2.62 \\
\hline hsa-miR-767-5p & 0.00002 & 4.94 & 2.31 \\
\hline HS_29 & 0.01811 & 4.41 & 2.14 \\
\hline hsa-miR-708 & 0.02645 & 3.92 & 1.97 \\
\hline hsa-miR-146a & 0.01076 & 3.84 & 1.94 \\
\hline hsa-miR-34a & 0.00268 & 3.13 & 1.65 \\
\hline solexa-539-2056 & 0.04379 & 3.03 & 1.60 \\
\hline hsa-miR-335* & 0.00486 & 2.81 & 1.49 \\
\hline hsa-miR-18b & 0.04529 & 2.79 & 1.48 \\
\hline hsa-miR-452* & 0.00000 & 2.58 & 1.37 \\
\hline hsa-miR-18a & 0.00441 & 2.56 & 1.36 \\
\hline hsa-miR-199a*:9.1 & 0.03622 & 2.56 & 1.36 \\
\hline hsa-miR-574-5p & 0.00000 & 2.39 & 1.26 \\
\hline hsa-miR-296-3p & 0.02907 & 2.36 & 1.24 \\
\hline hsa-miR-155 & 0.01372 & 2.33 & 1.22 \\
\hline hsa-miR-24-2* & 0.00000 & 2.33 & 1.22 \\
\hline hsa-miR-106a & 0.00149 & 2.32 & 1.21 \\
\hline hsa-miR-21* & 0.00073 & 2.22 & 1.15 \\
\hline HS_262.1 & 0.01814 & 2.16 & 1.11 \\
\hline hsa-miR-363 & 0.01021 & 2.07 & 1.05 \\
\hline hsa-miR-371-3p & 0.00002 & 2.03 & 1.02 \\
\hline \multicolumn{4}{|l|}{ b. } \\
\hline hsa-miR-129-3p & 0.00062 & -44.25 & -5.47 \\
\hline hsa-miR-129* & 0.00000 & -27.08 & -4.76 \\
\hline hsa-miR-1224-5p & 0.00515 & -16.54 & -4.05 \\
\hline hsa-miR-124a:9.1 & 0.00357 & -15.84 & -3.99 \\
\hline hsa-miR-326 & 0.01508 & -12.16 & -3.60 \\
\hline hsa-miR-124 & 0.00073 & -9.66 & -3.27 \\
\hline hsa-miR-204 & 0.00140 & -9.65 & -3.27 \\
\hline hsa-miR-1296 & 0.00000 & -6.94 & -2.80 \\
\hline hsa-miR-885-5p & 0.00006 & -6.76 & -2.76 \\
\hline hsa-miR-128a:9.1 & 0.00149 & -5.78 & -2.53 \\
\hline hsa-miR-218 & 0.00504 & -5.72 & -2.52 \\
\hline hsa-miR-133b & 0.00000 & -5.57 & -2.48 \\
\hline hsa-miR-874 & 0.00013 & -5.52 & -2.46 \\
\hline hsa-miR-769-3p & 0.02429 & -4.96 & -2.31 \\
\hline
\end{tabular}

Table 3 The top deregulated microRNAs in pilocytic astrocytoma compared to normal adult cerebellum (Continued)

\begin{tabular}{llll}
\hline hsa-miR-485-3p & 0.01589 & -4.70 & -2.23 \\
hsa-miR-128b:9.1 & 0.00049 & -4.68 & -2.23 \\
HS_182.1 & 0.00140 & -4.45 & -2.15 \\
hsa-miR-107 & 0.00002 & -4.38 & -2.13 \\
hsa-miR-383 & 0.00175 & -4.36 & -2.12 \\
hsa-miR-656 & 0.00825 & -4.35 & -2.12 \\
hsa-miR-124* & 0.03622 & -4.14 & -2.05 \\
hsa-miR-584 & 0.00130 & -3.61 & -1.85 \\
hsa-miR-7 & 0.00108 & -3.40 & -1.77 \\
hsa-miR-548k & 0.01014 & -3.39 & -1.76 \\
hsa-miR-1237 & 0.00149 & -3.21 & -1.68 \\
hsa-miR-299-5p & 0.01076 & -3.20 & -1.68 \\
hsa-miR-1179 & 0.01508 & -2.92 & -1.55 \\
hsa-miR-487b & 0.03622 & -2.75 & -1.46 \\
HS_231 & 0.00367 & -2.73 & -1.45 \\
hsa-miR-329 & 0.00101 & -2.47 & -1.30 \\
hsa-miR-154* & 0.00566 & -2.47 & -1.30 \\
hsa-miR-889 & 0.01811 & -2.15 & -1.11 \\
hsa-miR-135a & 0.02645 & -2.06 & -1.04 \\
\hline (a) Up-regulated microRNAs and (b) down-regulated microRNAs. Differential \\
expression was defined as fold change (FC) $>2$ with FDR corrected (Benjamini \\
Hochberg) $p$-values $<0.05$ & & & \\
& & &
\end{tabular}

Using TaqMan assays and Real Time qRT-PCR, we confirmed the differential expression of a set of microRNAs and genes in a selection of the test tumor cohort, as well as 13 tumors from the validation tumor cohort (Additional file 7: Figure S1 and Additional file 8: Figure S2). We confirmed up-regulation of miR-34a, miR-146a, miR-542-3p, miR-503 and miR-155 and low expression of neuronal microRNAs miR-124*, miR-129 and miR129* (Additional file 7: Figure S1). Up-regulation of the senescence factors IGFBP7, CDKN2A (p16), IL6, IL8, $C D K N 1 A$ ( $p 21)$ and down-regulation of $C T C F$ was also confirmed (Additional file 8: Figure S2).

\section{Discussion}

To gain a comprehensive understanding of tumorigenesis, it is critical to integrate findings on factors that regulate the transcriptome, such as microRNAs, with genomic analysis. This is especially pertinent in the case of pediatric CNS tumors, which have fewer mutational events than their adult counterparts [48, 54]. MicroRNAs are highly regulated and have essential functions in brain development $[23,30]$, and certain microRNAs are enriched or specifically expressed in the adult brain [49]. Here, we profiled microRNA and gene expression in a cohort of pediatric brain tumors, and then focused on the potential role of microRNAs in pilocytic astrocytomas. 


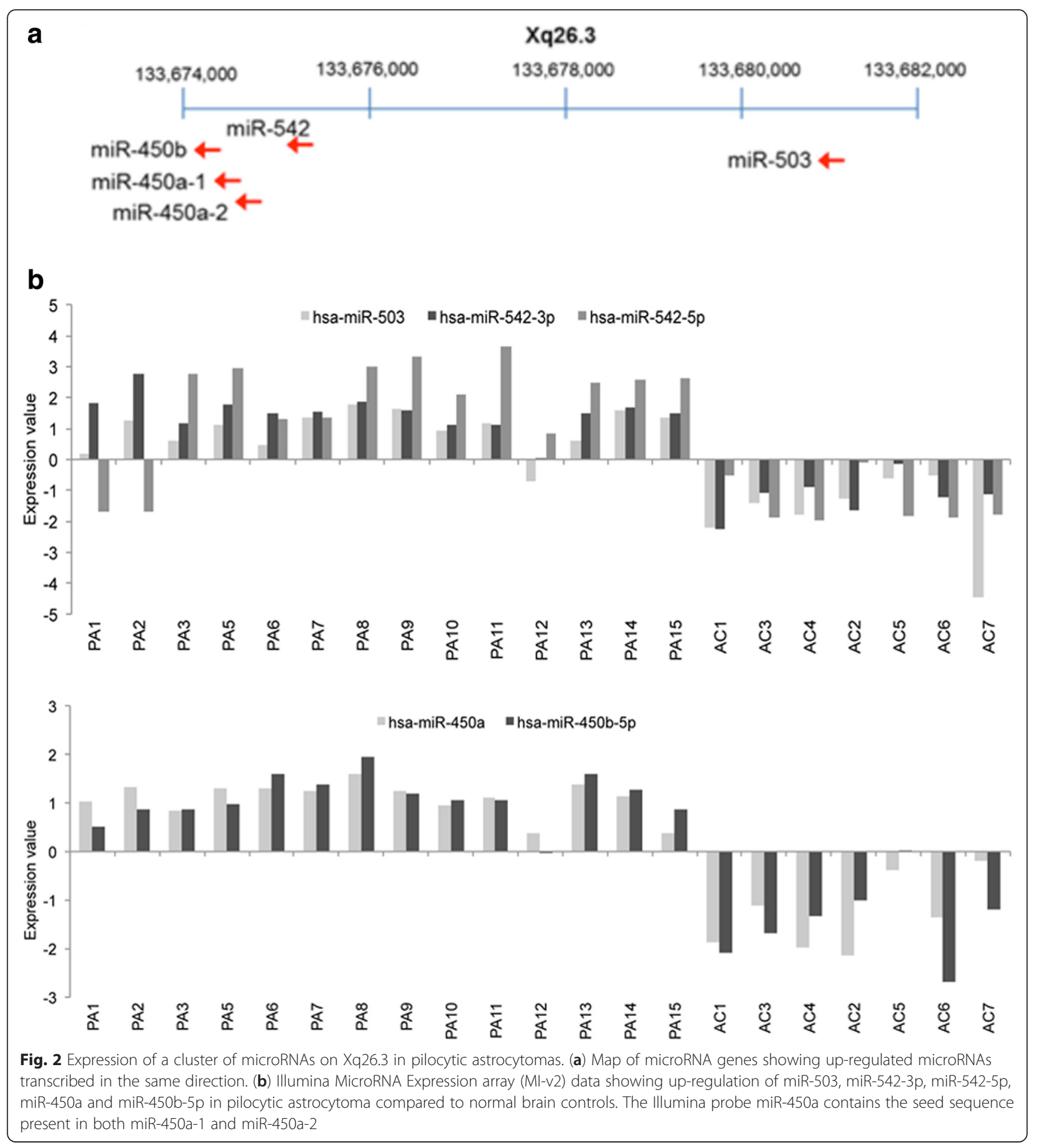

The mitogen-activated protein kinase (MAPK) signaling cascade is the main molecular pathway that is deregulated in pilocytic astrocytomas [56]. BRAF-gene fusions and, in rare cases, $B R A F$ V600E mutations give rise to constitutively active $B R A F$, which in turn activates the MAPK pathway. Furthermore, $B R A F$ activation has been shown to cause oncogene-induced senescence in vitro [24, 42]. Hence, we obtained gene expression profiles for pilocytic astrocytomas in order to identify any senescent or inflammatory markers that may explain the less aggressive nature of these tumors. Senescence is accompanied by increased secretion of factors involved in senescent signaling. In pilocytic astrocytomas, we observed up-regulation of IGFBP7 and $C E B P B$, both of which are major transcriptional inducers of SASP-related genes [16]. In addition, we observed up- 


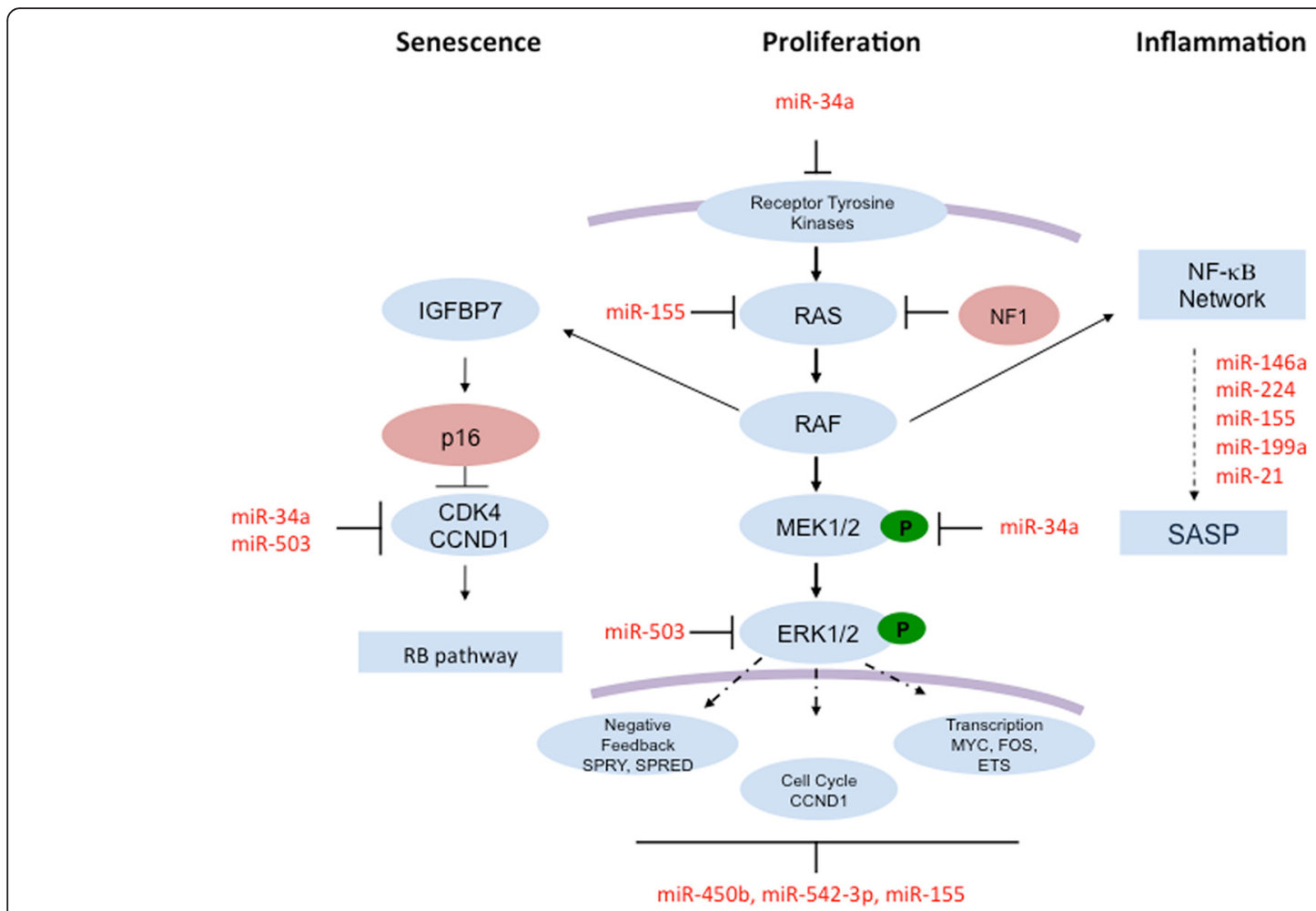

Fig. 3 Model showing the interplay of microRNAs in senescence, proliferation and inflammation. MicroRNAs provide negative feedback of the RB pathway, the MAPK pathway and the NF-KB network. MiR-34a and miR-503 are predicted to target CCND1 and CDK4 to limit cell proliferation via the RB pathway. MiR-155 is predicted to target KRAS, miR-34a is predicted to target MEK1, whilst down stream targets of the MAPK pathway include SPRED1 (a predicted target of miR-503), SPRY1, SPRY2 and SPRY4 (predicted targets of miR-450b-5p) and FOS and ETS1 (predicted targets of miR-155). MicroRNAs that act as effectors and regulators of NF-KB include miR-146a, miR-155, miR-199a and miR-21. MiR-146a, miR-224 and miR-155 are predicted to target senescenceassociated genes and thus to regulate levels of inflammation

regulation of $I L 1 B$, which has been shown to be a SASP-associated protein [10]. Our gene expression profiling of pilocytic astrocytomas also revealed up-regulation of many genes involved in immune function. This was supported by pathway analysis, which showed that pilocytic astrocytomas exhibit a strong immune and inflammatory response.

There is accumulating evidence for a role for certain microRNAs in senescence [1, 17, 19]. The miR-424miR-503 polycistron, as well as miR-450, miR-542-3p and miR-450b-5p are suggested to play a role in replicative senescence and cellular aging in fibroblasts [6]. MiR-424 and miR-503 can induce G1 arrest in THP-1 cells [14] and may limit proliferation in pilocytic astrocytomas. Recently, expression of miR-542-5p, which is repressed in $M Y C$-amplified neuroblastomas was shown to be positively correlated with survival [46]. Interestingly, CTCF, a predicted target of $\mathrm{miR}-542-3 p$, is down-regulated in pilocytic astrocytoma, and a recent investigation into the higher-order chromatin structure of the INK4/ARF locus in senescent cells, has revealed down-regulation of CTCF in oncogene-induced senescent cells [20].
Predicted targets of differentially regulated microRNAs frequently included components of the ERK/ MAPK and NF-kB signaling pathways (Fig. 3). MiR-503 and miR155 are predicted to target components of the ERK/MAPK pathway, as well as miR-34a which can induce cell cycle arrest by targeting Notch ligand deltalike 1, a component of NOTCH signaling [11], and cell cycle regulators CCND1 and CDK6 [52]. The same microRNAs are also predicted to target components of NF- $\mathrm{kB}$ signaling. MiR-155 has many target genes including $I K K \varepsilon$, suggesting a role in the immune response [53]. In ovarian tumors, miR-199a negatively regulates $I K K \beta$ expression to reduce $N F-\kappa B$ activity [8]. $N F-\kappa B$ is reported to be a direct transcriptional regulator of miR224 [47] and we observe up-regulation of the miR-224 in our pilocytic astrocytomas. In addition, miR-224 has been shown to target the tumor suppressor RKIP in human breast cancer [22]. MiR-146a, which is induced by inflammation in the presence of $N F-\kappa B$ and $I L 1$ receptor signaling to modulate senescent mediators, is upregulated specifically in pilocytic astrocytomas [4]. In fibroblasts, miR-146a/b down-regulates IRAK1 to reduce 


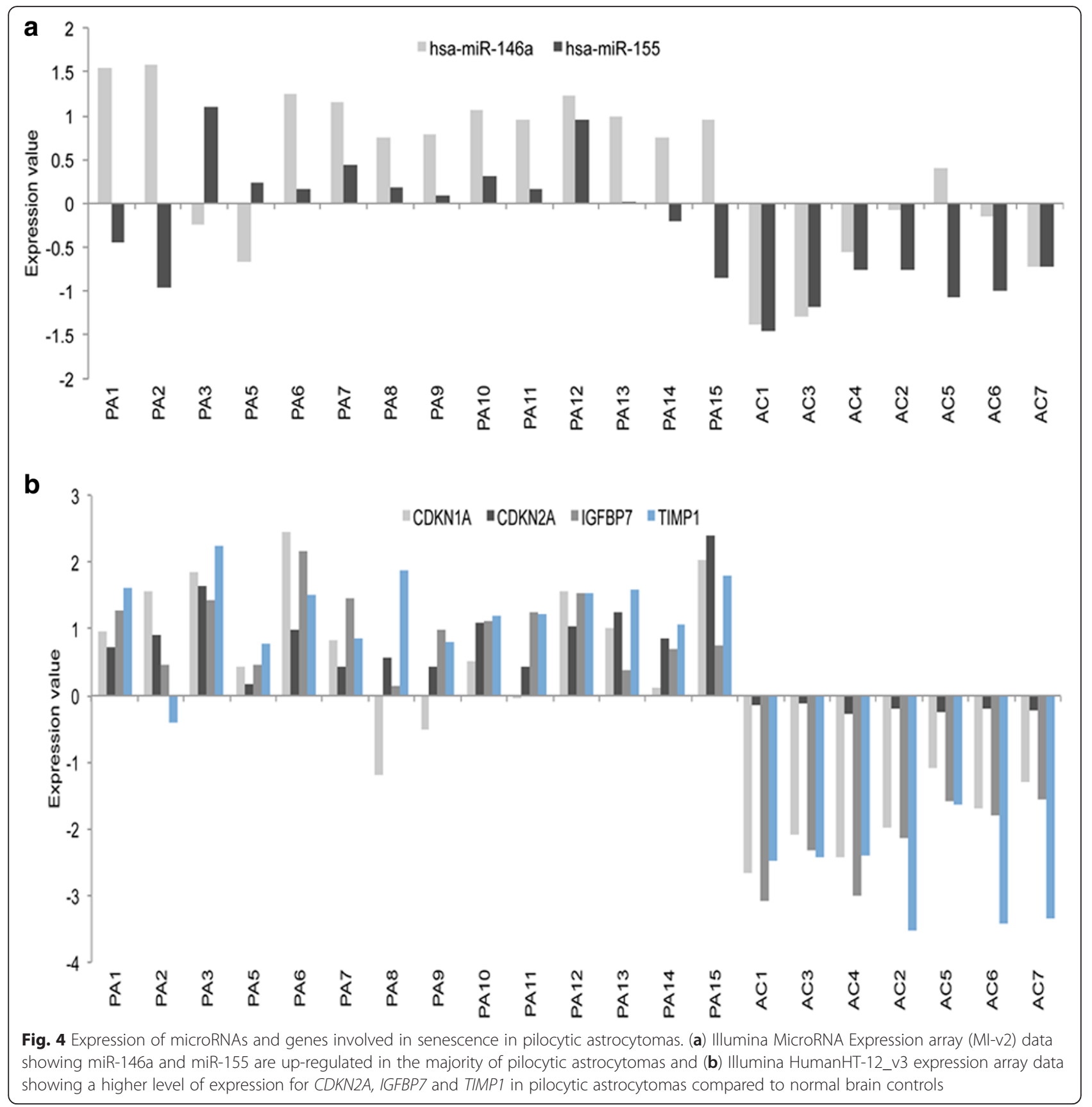

IL6 and IL8 secretion in an elegant feedback loop [4]. The expression of miR-146a is a late manifestation of senescence [43] and it is possible that the few pilocytic astrocytomas that do not show elevated expression of miR-146a have not reached a "mature" level of the senescent phenotype.

Brain tissues are composed of multiple neuronal, astroglial, microglial and other cell types, so the relative contributions of each brain cell type to the overall microRNA and gene expression profiles remain to be elucidated. MiR-146a and miR-155 are expressed by activated microglia and astroglial cells in culture [7, 34, 44]. Therefore, it is possible that some of our up-regulated microRNAs could also potentially represent the proliferating microglia that are reported to be present in pilocytic astrocytomas [28]. A recent study has demonstrated that increased activation of RAS/ERK signaling in neocortical progenitors produced a NEUROG2-ASCL1 switch, which promotes glial fate [55]. This developmental neurogenic to gliogenic switch in transcription factor expression is supported in studies of RAS-driven glioma formation from neural stem cells $[25,40]$. In a 
recently developed mouse model of gliomagenesis, high RAS/ERK levels were found to modify Ascll activity giving rise to glial tumors [33], and we observe clear up-regulation of ASCL1 in the pilocytic astrocytomas (Additional file 4: Table S4). These authors demonstrated that whilst RAS/ERK signaling is critical for neurodevelopment, ERK is the key effector as it phosphorylates the ASCL1 protein resulting in its gliogenic effect. Hence, enhanced RAS/ERK signaling in the pilocytic astrocytomas by activated BRAF may have triggered a gliogenic switch, and this is reflected in the expression of microRNAs that are usually observed in astroglial cells.

\section{Conclusions}

In summary, we show that pilocytic astrocytomas differentially express microRNAs that target genes that encode regulators of the MAPK and NF- $\mathrm{KB}$ pathways, as well as genes that are markers of inflammation. These microRNAs and the post-translational regulation of their gene targets may therefore play a role in the unique phenotype of pilocytic astrocytomas.

\section{Additional files}

\section{Additional file 1: Table S1. Normalized microRNA expression data. (XLSX $841 \mathrm{~kb})$}

Additional file 2: Table S2. Normalized gene expression data. (XLSX $37339 \mathrm{~kb})$

Additional file 3: Table S3. Differentially expressed microRNAs in pediatric brain tumors (tumor groups with > 3 samples). (XLSX 75 kb)

Additional file 4: Table S4. Differentially expressed genes in pediatric brain tumors (tumor groups with $>3$ samples). (XLSX 852 kb)

Additional file 5: Table S5. (a) List of primer pairs selected for Illumina microRNA expression array (MI-v2) validation. (b) List of primer pairs used to identify KIAA1549-BRAF fusion and the BRAF V600E mutation.

(PDF $58 \mathrm{~kb}$ )

Additional file 6: Table S6. Ingenuity Pathway Analysis of differentially expressed genes in pilocytic astrocytomas compared to normal adult cerebellum, showing transcription factors with a predicted activation state as (a) 'activated' and (b) 'inhibited'. (PDF 68 kb)

Additional file 7: Figure S1. RT-qPCR confirms (a) up-regulation of miR-34a, miR-146a, miR-542-3p and miR-503 in pilocytic astrocytomas. (b) low expression of miR-124*, miR-129 and miR-129* in pilocytic astrocytomas. Relative expression shown as $\log _{2}$ fold change compared to normal adult cerebellum and frontal lobe (normalized to miR-423-3p). Data represent two technical replicates \pm SD. (ZIP $516 \mathrm{~kb}$ )

Additional file 8: Figure S2. RT- $q P C R$ confirms (a) up-regulation of CDKN1A and IGFBP7, (b) up-regulation of CDKN2A, IL6 and IL8 and down-regulation of CTCF in pilocytic astrocytomas. Relative expression shown as $\log _{2}$ fold change compared to normal adult cerebellum and frontal lobe (normalized to TBP). Data represent two technical replicates \pm SD. (ZIP $275 \mathrm{~kb})$

\section{Competing interests}

The authors declare that they have no competing interests.

\section{Authors' contributions}

TAJ, JNJ, TF, RGT, ARJL, TSJ and DS conceived and designed the experiments. TAJ, JNJ, TF, RGT, ARJL, GTD and MAM performed the experiments, TAJ, JNJ,
TF, ARJL and SNP performed scientific analysis, TSJ conducted the pathological review. SRP, KPP, AM and TSJ provided scientific input. TAJ, JNJ and DS wrote the paper. TF, RGT, KPP, TSJ, TAJ, JNJ and DS revised the manuscript. All Authors read and approved the final manuscript.

\section{Acknowledgements}

This work was funded by The Brain Tumour Charity, Rosetrees Trust, Astro Brain Tumour Fund, Brain Tumour Action, and A Kid's Brain Tumor Cure (PLGA Foundation), and supported by the National Institute for Health Research Biomedical Research Centre at Great Ormond Street Hospital for Children NHS Foundation Trust and University College London. The funders had no role in the study design, data collection and analysis, decision to publish, or preparation of the manuscript. Primers for IL6, IL8 and CDKN2A (p16) were kindly provided by Dr. C. Bishop.

\section{Author details}

${ }^{1}$ Centre for Genomics and Child Health, Blizard Institute, Barts and The London School of Medicine and Dentistry, Queen Mary University of London, 4 Newark Street, London E1 2AT, UK. ²Department of Histopathology, Great Ormond Street Hospital for Children NHS Trust, London WC1N 3JH, UK. ${ }^{3}$ Developmental Biology and Cancer Programme, UCL-Institute of Child Health, University College London, London WC1N 1EH, UK. ${ }^{4}$ Department of Neurosurgery, Great Ormond Street Hospital for Children NHS Trust, London WC1N 3JH, UK. ${ }^{5}$ Department of Oncology, Great Ormond Street Hospital for Children NHS Trust, London WC1N 3JH, UK. ${ }^{6}$ Present address: Research Department of Pathology, Cancer Institute, University College London, 72 Huntley St, Camden Town, London WC1E 6DD, UK. PPresent address: Department of Neuropathology, St Jude Children's Research Hospital, Memphis, TN 38105, USA. ${ }^{8}$ Present address: Inivata Limited, Li Ka Shing Centre, Cambridge CB2 ORE, UK.

Received: 28 November 2015 Accepted: 5 December 2015

Published online: 18 December 2015

\section{References}

1. Abdelmohsen K, Srikantan S, Kang MJ, Gorospe M (2012) Regulation of senescence by microRNA biogenesis factors. Ageing Res Rev: Doi 10.1016/j. arr.2012.01.003

2. Ambros V, Bartel B, Bartel DP, Burge CB, Carrington JC, Chen $X$, et al. A uniform system for microRNA annotation. RNA. 2003;9:277-9.

3. Babashah S, Soleimani M. The oncogenic and tumor suppressive roles of microRNAs in cancer and apoptosis. Eur J Cancer. 2011;47:1127-37. doi:10.1016/j.ejca.2011.02.008.

4. Bhaumik D, Scott GK, Schokrpur S, Patil CK, Orjalo AV, Rodier F, et al. MicroRNAs miR-146a/b negatively modulate the senescence-associated inflammatory mediators IL-6 and IL-8. Aging (Albany NY). 2009;1:402-11.

5. Birks DK, Barton VN, Donson AM, Handler MH, Vibhakar R, Foreman NK. Survey of MicroRNA expression in pediatric brain tumors. Pediatr Blood Cancer. 2011:56:211-6. doi:10.1002/pbc.22723.

6. Bonifacio LN, Jarstfer MB (2010) MiRNA profile associated with replicative senescence, extended cell culture, and ectopic telomerase expression in human foreskin fibroblasts. PLoS One 5: Doi 10.1371/journal.pone.0012519

7. Cardoso AL, Guedes JR, Pereira de Almeida L, Pedroso de Lima MC. miR-155 modulates microglia-mediated immune response by down-regulating SOCS-1 and promoting cytokine and nitric oxide production. Immunology. 2012;135:73-88. doi:10.1111/j.1365-2567.2011.03514.x

8. Chen R, Alvero AB, Silasi DA, Kelly MG, Fest S, Visintin I, et al. Regulation of IKKbeta by miR-199a affects NF-kappaB activity in ovarian cancer cells. Oncogene. 2008;27:4712-23. doi:10.1038/onc.2008.112.

9. Collins VP, Jones DT, Giannini C. Pilocytic astrocytoma: pathology, molecular mechanisms and markers. Acta Neuropathol. 2015;129:775-88. doi:10.1007/s00401-015-1410-7.

10. Coppe JP, Patil CK, Rodier F, Sun Y, Munoz DP, Goldstein J, et al. Senescence-associated secretory phenotypes reveal cell-nonautonomous functions of oncogenic RAS and the p53 tumor suppressor. PLoS Biol. 2008; 6:2853-68. doi:10.1371/journal.pbio.0060301

11. de Antonellis P, Medaglia C, Cusanelli E, Andolfo I, Liguori L, De Vita G, et al. MiR-34a targeting of Notch ligand delta-like 1 impairs CD15+/CD133+ tumorpropagating cells and supports neural differentiation in medulloblastoma. PLoS One. 2011;6:e24584. doi:10.1371/journal.pone.0024584. 
12. Edgar R, Domrachev M, Lash AE. Gene Expression Omnibus: NCBI gene expression and hybridization array data repository. Nucleic Acids Res. 2002 30:207-10

13. Farrell BC, Power EM, Mc Dermott KW. Developmentally regulated expression of Sox9 and microRNAs 124, 128 and 23 in neuroepithelial stem cells in the developing spinal cord. Int J Dev Neurosci. 2011;29:31-6. doi:10.1016/j.ijdevneu. 2010.10.001.

14. Forrest AR, Kanamori-Katayama M, Tomaru $Y$, Lassmann $T$, Ninomiya $N_{\text {, }}$ Takahashi Y, et al. Induction of microRNAs, mir-155, mir-222, mir-424 and mir-503, promotes monocytic differentiation through combinatorial regulation. Leukemia. 2010;24:460-6. doi:10.1038/leu.2009.246.

15. Forshew T, Tatevossian RG, Lawson AR, Ma J, Neale G, Ogunkolade BW, et al. Activation of the ERK/MAPK pathway: a signature genetic defect in posterior fossa pilocytic astrocytomas. J Pathol. 2009;218:172-81. doi:10.1002/path.2558

16. Freund A, Orjalo AV, Desprez PY, Campisi J. Inflammatory networks during cellular senescence: causes and consequences. Trends Mol Med. 2010;16: 238-46. doi:10.1016/j.molmed.2010.03.003.

17. Gorospe M, Abdelmohsen K. MicroRegulators come of age in senescence. Trends Genet. 2011;27:233-41. doi:10.1016/j.tig.2011.03.005.

18. Griffiths-Jones S. The microRNA Registry. Nucleic Acids Res. 2004;32:D109-111. doi:10.1093/nar/gkh023.

19. He X, He L, Hannon GJ. The guardian's little helper: microRNAs in the p53 tumor suppressor network. Cancer Res. 2007;67:11099-101. doi:10.1158/0008-5472.CAN-07-2672.

20. Hirosue A, Ishihara K, Tokunaga K, Watanabe T, Saitoh N, Nakamoto M, et al. (2012) Quantitative assessment of higher-order chromatin structure of the INK4/ARF locus in human senescent cells. Aging Cell: doi:10.1111/j.14749726.2012.00809.x

21. Ho CY, Bar E, Giannini C, Marchionni L, Karajannis MA, Zagzag D, et al. MicroRNA profiling in pediatric pilocytic astrocytoma reveals biologically relevant targets, including PBX3, NFIB, and METAP2. Neuro Oncol. 2013;15: 69-82. doi:10.1093/neuonc/nos269.

22. Huang L, Dai T, Lin X, Zhao X, Chen X, Wang C, et al. MicroRNA-224 targets RKIP to control cell invasion and expression of metastasis genes in human breast cancer cells. Biochem Biophys Res Commun. 2012;425:127-33. doi:10.1016/j.bbrc.2012.07.025.

23. Im HI, Kenny PJ. MicroRNAs in neuronal function and dysfunction. Trends Neurosci. 2012;35:325-34. doi:10.1016/j.tins.2012.01.004.

24. Jacob K, Quang-Khuong DA, Jones DT, Witt H, Lambert S, Albrecht S, et al. Genetic aberrations leading to MAPK pathway activation mediate oncogene-induced senescence in sporadic pilocytic astrocytomas. Clin Cancer Res. 2011;17:4650-60. doi:10.1158/1078-0432.CCR-11-0127.

25. Jiang Y, Boije M, Westermark B, Uhrbom L. PDGF-B Can sustain selfrenewal and tumorigenicity of experimental glioma-derived cancerinitiating cells by preventing oligodendrocyte differentiation. Neoplasia. 2011:13:492-503.

26. Jonas S, Izaurralde E. Towards a molecular understanding of microRNAmediated gene silencing. Nat Rev Genet. 2015;16:421-33. doi:10.1038/nrg3965

27. Jones DT, Hutter B, Jager N, Korshunov A, Kool M, Warnatz HJ, et al. Recurrent somatic alterations of FGFR1 and NTRK2 in pilocytic astrocytoma. Nat Genet. 2013:45:927-32. doi:10.1038/ng.2682.

28. Klein R, Roggendorf W. Increased microglia proliferation separates pilocytic astrocytomas from diffuse astrocytomas: a double labeling study. Acta Neuropathol. 2001;101:245-8.

29. Kozomara A, Griffiths-Jones S. miRBase: annotating high confidence microRNAs using deep sequencing data. Nucleic Acids Res. 2014;42:D68-73. doi:10.1093/nar/gkt1181.

30. Krichevsky AM, King KS, Donahue CP, Khrapko K, Kosik KS. A microRNA array reveals extensive regulation of microRNAs during brain development. RNA. 2003;9:1274-81

31. Kuilman T, Michaloglou C, Vredeveld LC, Douma S, van Doorn R, Desmet CJ, et al. Oncogene-induced senescence relayed by an interleukin-dependent inflammatory network. Cell. 2008;133:1019-31. doi:10.1016/j.cell.2008.03.039.

32. Lawson AR, Tatevossian RG, Phipps KP, Picker SR, Michalski A, Sheer D, et al. RAF gene fusions are specific to pilocytic astrocytoma in a broad paediatric brain tumor cohort. Acta Neuropathol. 2010;120:271-3. doi:10.1007/s00401-010-0693-y.

33. Li S, Mattar P, Dixit R, Lawn SO, Wilkinson G, Kinch C, et al. RAS/ERK signaling controls proneural genetic programs in cortical development and gliomagenesis. J Neurosci. 2014;34:2169-90. doi:10.1523/JNEUROSCI. 4077-13.2014.

34. Li YY, Cui JG, Dua P, Pogue Al, Bhattacharjee S, Lukiw WJ. Differential expression of miRNA-146a-regulated inflammatory genes in human primary neural, astroglial and microglial cells. Neurosci Lett. 2011;499:109-13. doi:10.1016/j.neulet.2011.05.044.

35. Livak KJ, Schmittgen T. Analysis of relative gene expression data using realtime quantitative PCR and the 2(-Delta Delta C(T)) Method. Methods. 2001; 25:402-8. doi:10.1006/meth.2001.1262.

36. Louis DN, Ohgaki H, Wiestler OD, Cavenee WK, Burger PC, Jouvet A, et al. The 2007 WHO classification of tumors of the central nervous system. Acta Neuropathol. 2007;114:97-109. doi:10.1007/s00401-007-0243-4.

37. Melo SA, Esteller M. Dysregulation of microRNAs in cancer: playing with fire. FEBS Lett. 2011;585:2087-99. doi:10.1016/j.febslet.2010.08.009.

38. Miele E, Buttarelli FR, Arcella A, Begalli F, Garg N, Silvano M, et al. (2013) High-throughput microRNA profiling of pediatric high-grade gliomas. Neuro-oncology: Doi 10.1093/neuonc/not215

39. Ohgaki $H$, Kleihues P. Population-based studies on incidence, survival rates, and genetic alterations in astrocytic and oligodendroglial gliomas. J Neuropathol Exp Neurol. 2005;64:479-89.

40. Persson Al, Petritsch C, Swartling FJ, Itsara M, Sim FJ, Auvergne R, et al. Non-stem cell origin for oligodendroglioma. Cancer Cell. 2010;18:669-82. doi:10.1016/j.ccr.2010.10.033.

41. Qureshi IA, Mehler MF. Emerging roles of non-coding RNAs in brain evolution, development, plasticity and disease. Nat Rev Neurosci. 2012;13: 528-41. doi:10.1038/nrn3234

42. Raabe EH, Lim KS, Kim JM, Meeker A, Mao XG, Nikkhah G, et al. BRAF activation induces transformation and then senescence in human neural stem cells: a pilocytic astrocytoma model. Clin Cancer Res. 2011;17:3590-9. doi:10.1158/1078-0432.CCR-10-3349.

43. Rodier F, Campisi J. Four faces of cellular senescence. J Cell Biol. 2011;192: 547-56. doi:10.1083/jcb.201009094.

44. Saba R, Gushue S, Huzarewich RL, Manguiat K, Medina S, Robertson C, et al. MicroRNA 146a (miR-146a) is over-expressed during prion disease and modulates the innate immune response and the microglial activation state. PLoS One. 2012;7:e30832. doi:10.1371/journal.pone.0030832.

45. Schindler G, Capper D, Meyer J, Janzarik W, Omran H, Herold-Mende C, et al Analysis of BRAF V600E mutation in 1,320 nervous system tumors reveals high mutation frequencies in pleomorphic xanthoastrocytoma, ganglioglioma and extra-cerebellar pilocytic astrocytoma. Acta Neuropathol. 2011;121:397-405. doi:10.1007/s00401-011-0802-6.

46. Schulte $\mathrm{JH}$, Schowe B, Mestdagh $\mathrm{P}$, Kaderali L, Kalaghatgi $\mathrm{P}$, Schlierf S, et al. Accurate prediction of neuroblastoma outcome based on miRNA expression profiles. Int J Cancer. 2010;127:2374-85. doi:10.1002/ijc.25436.

47. Scisciani C, Vossio S, Guerrieri F, Schinzari V, De laco R, D'Onorio De Meo P, et al. Transcriptional regulation of miR-224 upregulated in human HCCs by NFkappaB inflammatory pathways. J Hepatol. 2012;56:855-61. doi:10.1016/j.jhep.2011.11.017.

48. Scotting PJ, Walker DA, Perilongo G. Childhood solid tumors: a developmental disorder. Nat Rev Cancer. 2005;5:481-8. doi:10.1038/nrc1633.

49. Sempere LF, Freemantle S, Pitha-Rowe I, Moss E, Dmitrovsky E, Ambros $\checkmark$. Expression profiling of mammalian microRNAs uncovers a subset of brain-expressed microRNAs with possible roles in murine and human neuronal differentiation. Genome Biol. 2004;5:R13. doi:10.1186/gb-2004-5-3-r13gb-2004-5-3-r13.

50. Smirnova L, Grafe A, Seiler A, Schumacher S, Nitsch R, Wulczyn FG. Regulation of miRNA expression during neural cell specification. Eur J Neurosci. 2005;21:1469-77. doi:10.1111/j.1460-9568.2005.03978.x.

51. Sosanya NM, Huang PP, Cacheaux LP, Chen CJ, Nguyen K, Perrone-Bizzozero NI, et al. Degradation of high affinity HuD targets releases Kv1.1 mRNA from miR-129 repression by mTORC1. J Cell Biol. 2013;202:53-69. doi:10.1083/jcb.201212089.

52. Sun F, Fu H, Liu Q, Tie Y, Zhu J, Xing R, et al. Downregulation of CCND1 and CDK6 by miR-34a induces cell cycle arrest. FEBS Lett. 2008;582:1564-8. doi:10.1016/j.febslet.2008.03.057.

53. Tili E, Michaille JJ, Cimino A, Costinean S, Dumitru CD, Adair B, et al. Modulation of miR-155 and miR-125b levels following lipopolysaccharide/ TNF-alpha stimulation and their possible roles in regulating the response to endotoxin shock. J Immunol. 2007;179:5082-9.

54. Vogelstein B, Papadopoulos N, Velculescu VE, Zhou S, Diaz Jr LA, Kinzler KW. Cancer genome landscapes. Science. 2013;339:1546-58. doi:10.1126/science.1235122. 
55. Wang Y, Kim E, Wang X, Novitch BG, Yoshikawa K, Chang LS, et al. ERK inhibition rescues defects in fate specification of Nf1-deficient neura progenitors and brain abnormalities. Cell. 2012;150:816-30. doi:10.1016/j.cell.2012.06.034

56. Zhang J, Wu G, Miller CP, Tatevossian RG, Dalton JD, Tang B, et al. Whole-genome sequencing identifies genetic alterations in pediatric lowgrade gliomas. Nat Genet. 2013;45:602-12. doi:10.1038/ng.2611.

Submit your next manuscript to BioMed Central and we will help you at every step:

- We accept pre-submission inquiries

- Our selector tool helps you to find the most relevant journal

- We provide round the clock customer support

- Convenient online submission

- Thorough peer review

- Inclusion in PubMed and all major indexing services

- Maximum visibility for your research

Submit your manuscript at www.biomedcentral.com/submit 